\title{
Heterodimeric interaction between GKN2 and TFF1 entails synergistic antiproliferative and pro-apoptotic effects on gastric cancer cells
}

\author{
Olga Kim ${ }^{1} \cdot$ Jung Hwan Yoon ${ }^{1}$ Won Suk Choi ${ }^{1} \cdot$ Hassan Ashktorab ${ }^{2} \cdot$ \\ Duane T. Smoot ${ }^{2} \cdot$ Suk Woo Nam ${ }^{1} \cdot$ Jung Young Lee ${ }^{1} \cdot$ Won Sang Park ${ }^{1}$
}

Received: 17 August 2016/Accepted: 14 January 2017/Published online: 1 February 2017

(c) The International Gastric Cancer Association and The Japanese Gastric Cancer Association 2017

\begin{abstract}
Background GKN2 and TFF1 form a heterodimer that is only generated in the mucus-secreting cells of the normal stomach. The formation of this heterodimer is frequently disrupted in gastric cancer. However, the precise roles of GKN2 alone and in the heterodimer with TFF1 as well as the contributions of GKN2 and the heterodimer to gastric carcinogenesis are poorly understood.

Methods Cell viability, proliferation, and apoptosis were analyzed in AGS, MKN1, MKN28, and MKN45 gastric cancer cells transfected with GKN2 and/or TFF1 using MTT, BrdU incorporation, and apoptosis assays, respectively. In addition, cell viability was examined in HFE-145 non-neoplastic gastric epithelial cells after $G K N 2$ and/or TFF1 silencing. Furthermore, the cell cycle and the expression of cell cycle and apoptosis related proteins were assessed. The interaction between GKN2 and TFF1 was confirmed by co-immunoprecipitation. Immunohistochemistry was employed to explore TFF1 expression in 169 gastric cancer tissues.

Results Co-transfection with GKN2 and TFF1 significantly inhibited cell viability and proliferation by inducing G1/S cell cycle arrest and suppressing positive cell cycle
\end{abstract}

Electronic supplementary material The online version of this article (doi:10.1007/s10120-017-0692-y) contains supplementary material, which is available to authorized users.

Won Sang Park

wonsang@catholic.ac.kr

1 Department of Pathology, College of Medicine, The Catholic University of Korea, 505 Banpo-dong, Seocho-gu, Seoul 137-701, Korea

2 Department of Medicine, Howard University, Washington, DC 20060, USA regulators. Simultaneous knockdown of GKN2 and TFF1 in HFE-145 cells resulted in markedly increased cell viability. Moreover, the interaction of GKN2 and TFF1 promoted cell death by enhancing caspase-3/7 activity and upregulating pro-apoptotic proteins. At the mRNA level, GKN2 and TFF1 were found to be positively correlated in non-tumor and tumor samples. Immunohistochemistry revealed loss of TFF1 expression in 128 (75.73\%) of 169 gastric cancers. There was a borderline-significant association between GKN2 and TFF1 protein expression in gastric cancers $(P=0.0598)$.

Conclusion Collectively, our data demonstrated that the interaction between GKN2 and TFF1 can have synergistic antiproliferative and pro-apoptotic effects on gastric cancer.

Keywords GKN2 - TFF1 - Proliferation · Apoptosis · Gastric cancer

\section{Introduction}

Despite numerous efforts to improve treatment efficacy and increase survival rates, gastric cancer remains a global burden, as it is one of the leading causes of cancer mortality worldwide [1].

TFF1 (trefoil factor family 1; encoded by the TFF1 gene, also known as $p S 2$ ) was originally identified as an estrogen-regulated mRNA in breast cancer cells $[2,3]$. Further studies have revealed that it is a gastric-specific peptide normally secreted predominantly in the foveolar and superficial epithelia of gastric mucosa [4]. TFF1 belongs to the trefoil factor family of proteins, which play essential roles in epithelial restitution and mucosal repair within the gastrointestinal tract [5]. Indeed, TFF1 has been 
found to be upregulated in response to mucosal injury induced by peptic ulceration [6,7], inflammatory bowel disease [8], and nonsteroidal anti-inflammatory drugs or ethanol [9]. Interestingly, TFF1 knockout mice exhibit severe hyperplasia and dysplasia, leading to adenomas and multifocal invasive carcinomas in $30 \%$ of the knockout mice [10]. In humans, there is a progressive loss of TFF1 from normal gastric mucosa to carcinoma, with only $50 \%$ of gastric cancers expressing TFF1 [11]. Somatic TFF1 inactivation, including mutation, allelic loss, and promoter methylation, is commonly detected in gastric cancers [12-14]. However, the molecular mechanisms responsible for the tumor-suppressive activities of TFF1 have not been fully clarified.

In a previous study, we showed that gastrokine 2 (GKN2) is able to inhibit the activity of GKN1, thereby contributing to gastric mucosal homeostasis [15]. Interestingly, GKN2 and TFF1 can form a heterodimer that is stabilized through an intermolecular disulfide bond between cysteine residues in the carboxy terminus of TFF1 (Cys58) and in the BRICHOS domain of GKN2 (Cys38) [16]. Notably, this heterodimer forms only in the mucussecreting cells of the stomach $[16,17]$. However, co-expression of GKN2 and TFF1 is frequently disrupted in gastric cancers and the expression of TFF1 in the absence of GKN2 is associated with a more invasive and metastatic phenotype [18]. It seems that GKN2, when acting as a protein partner to TFF1, may be involved in the regulation of important anticarcinogenic effects of TFF1. Although GKN2 has been identified as a gastric tumor suppressor $[19,20]$, the precise roles of GKN2 alone and when interacting with TFF1 are poorly understood.

Therefore, the objective of this study was to determine the effects of the interaction of GKN2 and TFF1 on gastric cancer cell viability, proliferation, and apoptosis. We hypothesized that GKN2 and TFF1 may possess synergistic antitumor activities in gastric cancer. Overall, we found that GKN2 and TFF1 heterodimer formation demonstrated substantial antiproliferative and pro-apoptotic effects, suggesting that TFF1 and GKN2 act synergistically to inhibit gastric carcinogenesis.

\section{Materials and methods}

\section{Cell culture and transfection with $G K N 2$ and $T F F 1$}

The cancer cell lines AGS, MKN1, MKN28, and MKN45 were obtained from the American Type Culture Collection and HFE-145 non-neoplastic gastric epithelial cells were obtained from Dr. Ashktorab and Dr. Smoot (Washington, DC, USA). The cells were cultured at $37{ }^{\circ} \mathrm{C}$ in $5 \% \mathrm{CO}_{2}$ in RPMI-1640 medium (Lonza, Basel, Switzerland) supplemented with $10 \%$ heat-inactivated fetal bovine serum. GKN2 and TFF1 cDNAs were cloned into the expression vector pcDNA3.1 (Invitrogen, Carlsbad, CA, USA). AGS, MKN1, MKN28, and MKN45 cells were transfected in 60-mm diameter dishes with expression plasmids $(2 \mu \mathrm{g}$ total DNA) using Lipofectamine Plus transfection reagent (Invitrogen, Carlsbad, CA, USA) according to the manufacturer's recommendations. The experimental groups were as follows: (1) mock control (transfection with empty vector); (2) transfection with GKN2; (3) transfection with TFF1; (4) co-transfection with GKN2 and TFF1.

\section{Measurement of cell viability and proliferation}

We investigated whether GKN2 and TFF1 are involved in the regulation of cell viability by performing MTT [3-(4,5dimethylthiazol-2-yl)-2,5-diphenyl-tetrazolium bromide] assays in AGS, MKN1, MKN28, and MKN45 cells after transfection with GKN2 and TFF1. The MTT assay was also performed in HFE-145 non-neoplastic gastric epithelial cells after silencing GKN2 and TFF1 by siGKN2 and siTFF 1, in order to further verify whether cell viability is dependent on the activities of the GKN2 and TFF1 proteins. Both siGKN2 and siTFF1 were purchased from Santa Cruz Biotechnology (Santa Cruz, CA, USA). Absorbance was measured with a spectrophotometer at $560 \mathrm{~nm}$, and cell viability was expressed relative to the mock control.

For cell proliferation analysis, a BrdU incorporation assay was performed in the AGS and MKN1 cells after transfection with GKN2 and TFF1 using the BrdU cell proliferation assay kit (Millipore, Billerica, MA, USA) according to the manufacturer's instructions. Absorbance was measured with a spectrophotometer at $450 \mathrm{~nm}$, and proliferation was expressed relative to the mock control.

\section{Co-immunoprecipitation (Co-IP)}

GKN2- and/or TFF1-transfected AGS and MKN1 cells were washed with PBS and lysed at $4{ }^{\circ} \mathrm{C}$ with PBS, pH 7.2, containing $1.0 \%$ NP-40, $0.5 \%$ sodium deoxycholate, $0.1 \%$ SDS, $10 \mathrm{mM} \mathrm{NaF}, 1.0 \mathrm{mM} \mathrm{NaVO}$, and $1.0 \%$ protease inhibitor cocktail (Sigma, St. Louis, MO, USA), as described previously [21]. Equal protein aliquots $(1.0 \mathrm{mg})$ were immunoprecipitated with $2.0 \mu \mathrm{g}$ of antibodies to GKN2 (Abcam, Cambridge, UK) and TFF1 (Santa Cruz Biotechnology) in protein A/G plus agarose (Santa Cruz Biotechnology), as described previously [22].

\section{Cell cycle analysis using flow cytometry}

We also explored the effects of GKN2 and TFF1 on cell cycle progression by flow cytometry. AGS and MKN1 cells 
from each experimental group were collected and stained with propidium iodide (PI) for $45 \mathrm{~min}$ in the dark prior to analysis. The percentages of cells at different phases of the cell cycle were determined using a BD FACS Canto II flow cytometer with FACSDiva software 8.0.1 (BD Biosciences, Heidelberg, Germany). Experiments were performed in triplicate, and the average values were used for quantification.

\section{Examination of the expression of cell cycle regulators by western blot analysis}

Further, we determined whether GKN2 and TFF1 influence the expression of cell cycle regulators. Expression of the G0/G1 and G2/M phase proteins, including p53, p21, CDK6, cyclin D1, E, A, and B, was examined in AGS and MKN1 cells at $72 \mathrm{~h}$ after transfection with $G K N 2$ and/or TFF1 by western blot analysis, as described previously [15]. The following antibodies were used: p53, p21, GAPDH (Santa Cruz Biotechnology), CDK6, cyclin D1, E, A, and B (Cell Signaling, Danvers, MA, USA).

\section{Measurement of apoptosis}

Apoptosis was measured using an annexin $\mathrm{V}$ binding assay according to the manufacturer's instructions (BD Biosciences). Annexin $\mathrm{V}$ binds to cells that display phosphatidylserine on the cell membrane, whereas PI stains cellular DNA with a compromised cell membrane. Transfected AGS, MKN1, MKN28, and MKN45 cells were washed twice in cold PBS and resuspended in $100 \mu \mathrm{l}$ binding buffer. In each case, $100 \mathrm{ml}$ supernatant were mixed with $400 \mu \mathrm{l}$ blocking solution and $5 \mu \mathrm{l}$ annexin V-FITC $(1 \mu \mathrm{g} / \mathrm{ml}), 5 \mu \mathrm{l} \mathrm{PI}(2 \mu \mathrm{g} / \mathrm{ml})$ were added, and the mixture was incubated in the dark for $15 \mathrm{~min}$. Cells were analyzed using a fluorescence-activated cell sorter (BD Biosciences). Annexin-V-positive cells without PI staining were considered to be apoptotic cells.

We also examined caspase- 3 and -7 activity with the Apo-One Homogeneous Caspase 3/7 assay kit (Promega Corporation, Madison, WI, USA), as described previously [23]. Expression of apoptosis-related proteins was also examined by western blot analysis in AGS and MKN1 cells transfected with GKN2 and/or TFF1. Briefly, cell lysates were separated on a $10 \%$ polyacrylamide gel and transferred onto a Hybond PVDF membrane (Bio-Rad, Richmond, CA, USA). After blocking, the membrane was probed with antibodies against the following proteins: Mcl1, BAX, Bcl-2, caspase-3, cleaved caspase-3, PARP, cleaved PARP (Cell Signaling), GAPDH (Santa Cruz Biotechnology), and mouse IgG and rabbit IgG (Sigma). An enhanced chemiluminescence plus western blotting detection system (Amersham Pharmacia Biotech,
Table 1 Primer sequences for real-time RT-PCR

\begin{tabular}{ll}
\hline Gene & Primer sequences \\
\hline GKN2 & F: $5^{\prime}$-TGCAGGATCATGCTCTTCTAC-3' \\
& R: $5^{\prime}$-TGGTCCATCTTCAGGATAAAG-3' \\
FFF1 & F: 5'-CCATGGCCACCATGGAGAAC-3' \\
& R: 5'-AACGGTGTCGTCGAAACAGC-3' \\
GAPDH & F: 5'-AAATCAAGTGGGGCGATGCTG-3' \\
& R: 5'-GCAGAGATGATGACCCTTTTG-3' \\
\hline
\end{tabular}

Piscataway, NJ, USA) was used to detect bound antibodies. The intensity of bands was quantified using LAS 3000 (Fujifilm, Tokyo, Japan).

Analysis of GKN2 and TFF1 expression in gastric
non-tumor and tumor tissues by real-time RT-PCR

Expression of GKN2 and TFF1 mRNAs was analyzed in 46 gastric cancer and corresponding non-tumor tissues by realtime RT-PCR. The primer sequences are listed in Table 1. Quantitative RT-PCR was performed on an IQ5 optical system (Bio-Rad) using SYBR Green Q-PCR Master Mix (Bio-Rad), as described previously [22]. All samples were tested in duplicate. Data are reported as relative quantities according to an internal calibrator using the $2^{-\Delta \Delta C T}$ method [24]. Written informed consent was obtained from all participants in accordance with the Declaration of Helsinki. The study was approved by the Institutional Review Board of The Catholic University of Korea, College of Medicine (CUMC09U089).

\section{Investigation of TFF1 expression in gastric cancers by immunohistochemistry}

Tissue microarray recipient blocks containing 169 gastric cancer tissues were constructed from formalin-fixed paraffin embedded specimens according to established methods [25]. One cylinder of normal gastric mucosa adjacent to each tumor was also transferred to the recipient block. Sections ( $2 \mu \mathrm{m}$ thick) were cut the day before use and stained according to the standard protocol.

To enhance the signal, we used antigen retrieval in citrate buffer and signal amplification with biotinylated tyramide [12]. The sections were incubated overnight at $4{ }^{\circ} \mathrm{C}$ with $\mathrm{TFF} 1$ antibodies (1/100; Santa Cruz Biotechnology), as described previously [15]. Staining for TFF1 antigen was considered positive when $>10 \%$ of the cancer cells showed positive staining. The results were reviewed independently by two pathologists. For negative controls, primary antibodies were replaced with non-immune serum. We compared TFF1 expression patterns with our previous GKN2 expression data [15]. 


\section{Statistical analysis}

The effects of GKN2 and TFF1 on cell viability, proliferation, the cell cycle, and apoptosis were assessed by Student's $t$ test. Data are expressed as the mean \pm SD from at least three independent experiments. Spearman's correlation and chi-square tests were used to investigate relationships between GKN2, TFF1, and clinicopathologic parameters. Statistical analyses were performed using Graphpad $^{\mathrm{TM}} 5.0$ (GraphPad Software Inc., San Diego, CA, USA). $P$ values $<0.05$ were considered statistically significant.

\section{Results}

\section{Heterodimeric interaction between GKN2 and TFF1}

Previously, Westley et al. immunopurified GKN2 using a TFF1-specific antibody from human gastric mucosal cells and showed that GKN2 is present with TFF1 in a heterodimer $[16,18]$. Therefore, we verified the interaction between GKN2 and TFF1 using co-immunoprecipitation analysis. GKN2 and TFF1 did indeed form a complex after co-transfection with GKN2 and TFF1 in AGS and MKN1 cells (Fig. 1a). Additional examination of whole-cell lysate served as a control for GKN2 and TFF1 protein expression (Fig. 1a).

\section{GKN2 and TFF1 synergistically inhibit cell viability and proliferation}

Cell viability and proliferation assays were performed in AGS, MKN1, MKN28, and MKN45 cells transfected with $G K N 2, T F F 1$, and $G K N 2$ plus TFF1. Transfection with TFF1 resulted in a considerable time-dependent decrease in cell viability, especially at 48 and $72 \mathrm{~h}$ after transfection (both $P<0.0001$ ), whereas $G K N 2$ alone led to minimal changes in cell viability (Fig. 1b, Supplementary Figure S1 in the Electronic supplementary material, ESM). Interestingly, co-transfection with both GKN2 and TFF1 stimulated a dramatic reduction in cell viability, which was rather more significant than transfection with either TFF1 or $G K N 2$ alone $(P<0.0001$, Fig. 1b, Supplementary Figure $\mathrm{S} 1$ in the ESM).

Additionally, we verified the effects of GKN2 and TFF1 on cell viability in HFE-145 non-neoplastic gastric epithelial cells after silencing GKN2 and TFF1 using siGKN2 and siTFF1 (Fig. 1d). Silencing GKN2 using siGKN2 did not lead to significant changes in cell viability, whereas knockdown of TFF1 resulted in elevated cell viability $\quad(P<0.05) . \quad$ Furthermore, simultaneous
Fig. 1a-d Synergistic antiproliferative effects of the interaction of GKN2 and TFF1 in gastric cancer cells. a In co-immunoprecipitation analysis, AGS and MKN1 cells transfected with GKN2 and TFF1 showed the formation of a complex of GKN2 and TFF1. Examination of whole-cell lysate served as a control for GKN2 and TFF1 protein expression. b In the MTT assay, TFF1 significantly suppressed cell viability in a time-dependent manner, whereas GKN2 alone failed to exert a notable effect on cell viability. The interaction of GKN2 and TFF1 synergistically enhanced the inhibition of the cell viability of AGS and MKN1 cells. $\mathbf{c}$ In the BrdU incorporation assay, transient transfection with TFF1 led to a time-dependent reduction in cell proliferation, especially at 48 and $72 \mathrm{~h}$ after transfection. However, co-expression of GKN2 and TFF1 resulted in a more substantial antiproliferative effect on AGS and MKN1 gastric cancer cells than transfection with $T F F 1$ or GKN1 alone. d Cell viability was examined by MTT assay in HFE-145 non-neoplastic gastric epithelial cells after silencing $G K N 2$ and $T F F 1$ using siGKN2 and siTFF1, respectively. $G K N 2$ silencing by siGKN2 did not lead to significant changes in cell viability, whereas knockdown of TFF1 resulted in elevated cell viability. Simultaneous knockdown of both $G K N 2$ and $T F F 1$ led to a more substantial increase in cell viability than silencing $G K N 2$ or TFF1 alone did. Data are expressed as mean values \pm SD from at least three independent experiments. The unpaired Student's $t$ test was used to analyze statistical differences in cell viability and proliferation between experimental groups. $P<0.05$ was considered statistically significant

knockdown of both GKN2 and TFF1 led to a more substantial increase in cell viability than silencing either $G K N 2$ or TFF1 alone $(P<0.05)$ (Fig. 1d).

In the BrdU incorporation assay, GKN2 expression failed to show any notable effect on cell proliferation. However, TFF1 expression led to a time-dependent reduction in cell proliferation at 48 and $72 \mathrm{~h}$ after transfection (both $P<0.0001$, Fig. 1c). Similarly, GKN2 and TFF1 co-expression resulted in considerably diminished proliferation levels of AGS and MKN1 cells (both $P<0.0001$, Fig. 1c).

\section{GKN2 and TFF1 co-expression restricts cell cycle progression by inducing G1/S arrest}

Next, we determined whether GKN2 and TFF1 are involved in cell cycle regulation. As shown in Fig. 2a, GKN2 alone had no effect on the cell cycle. However, TFF1 alone significantly increased the percentage of cells at the G1 phase while decreasing the number of cells at the $S$ phase. In addition, the interaction between GKN2 and TFF1 triggered G1/S cell cycle arrest in both AGS and MKN1 cells (Fig. 2a).

Therefore, we checked whether GKN2 and TFF1 could modulate cell cycle progression by regulating G0-, G1/S-, and G2/M-phase-related proteins. Western blot analysis revealed markedly elevated expression levels of p53 and p21 in cells transfected with TFF1 or with GKN2 plus TFF1 (Fig. 2b). Furthermore, the interaction between GKN2 and TFF1 considerably downregulated the 
a

AGS

MKN1

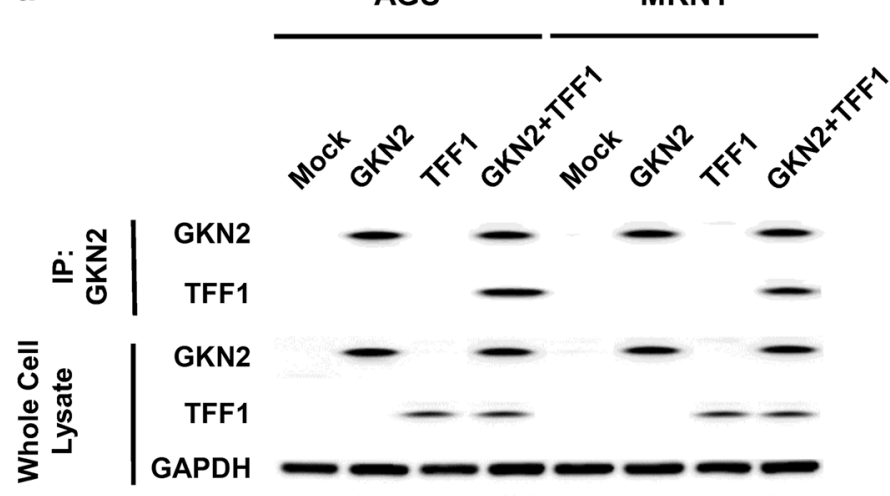

b
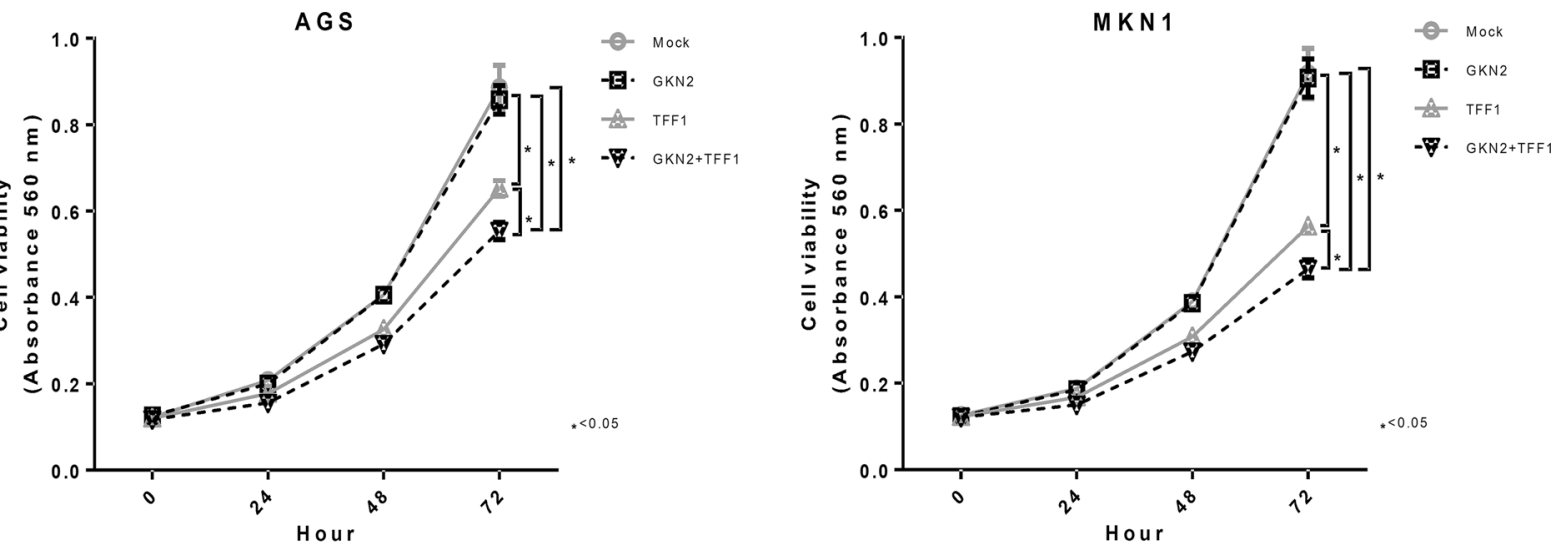

c
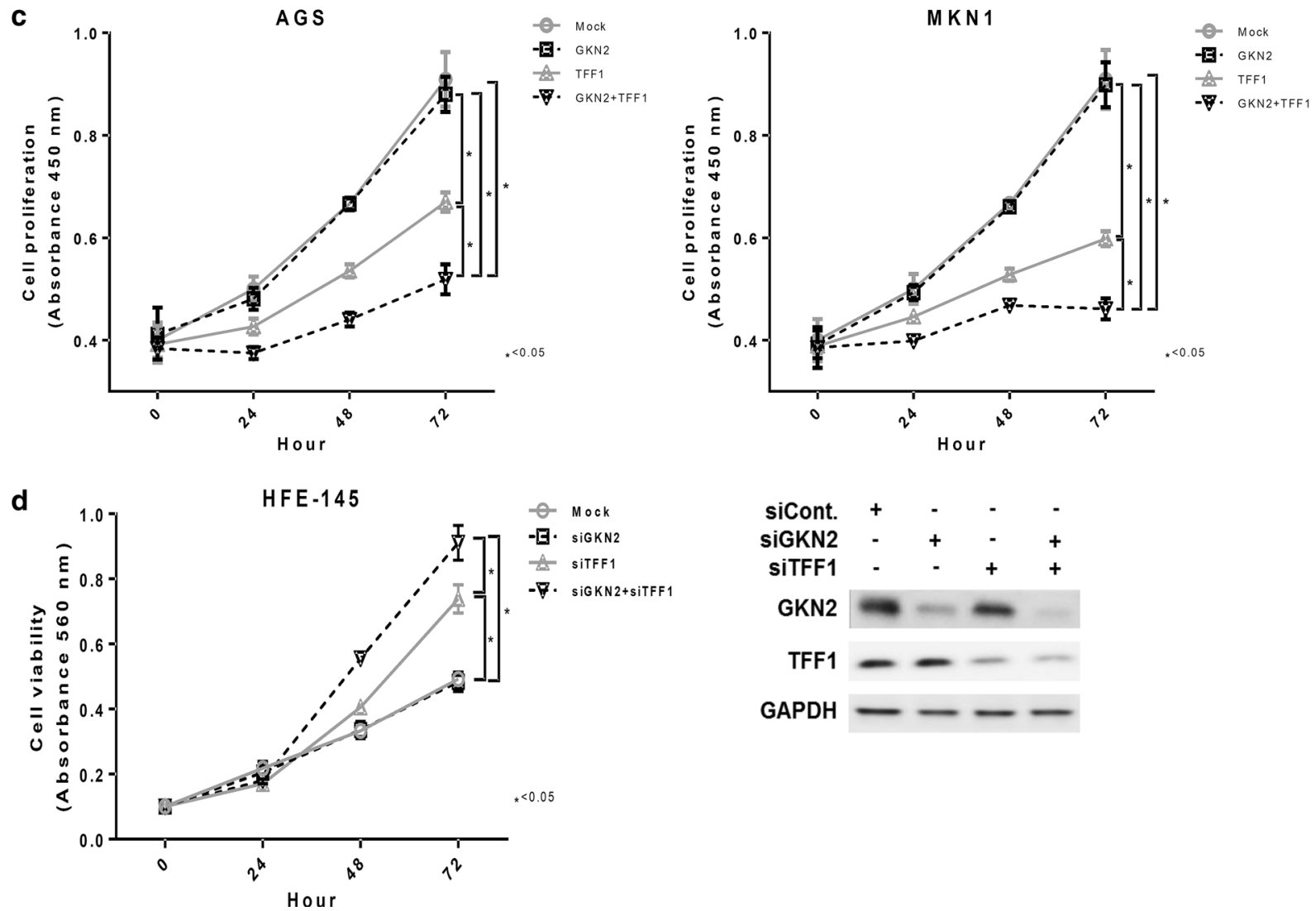
a

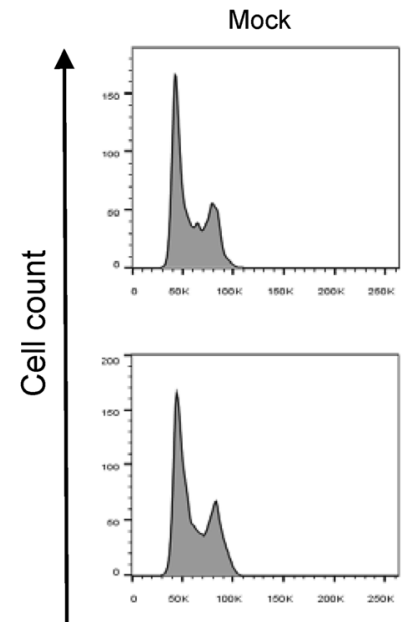

GKN2
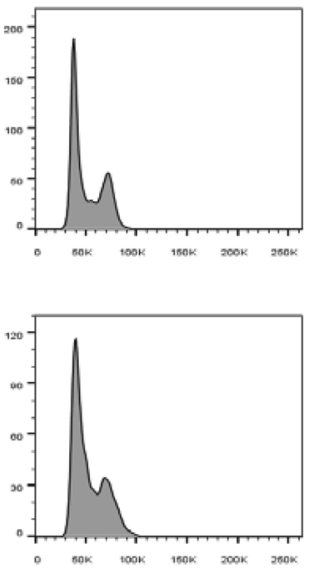

TFF1
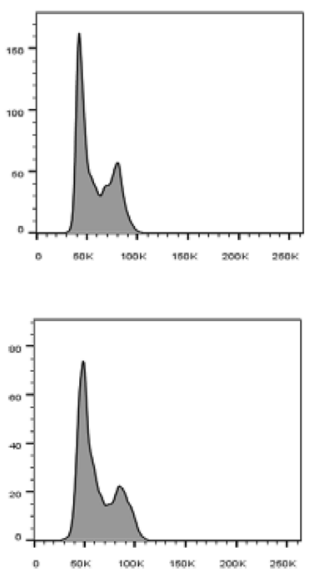

GKN2+TFF1

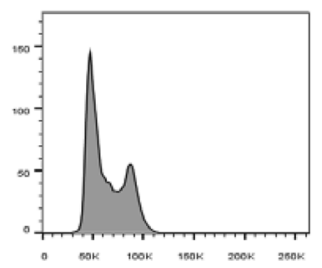

AGS

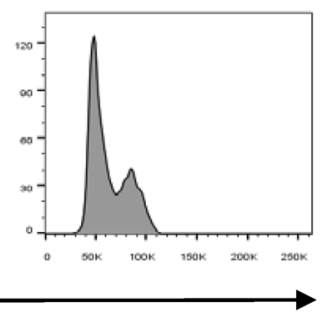

MKN1

DNA content

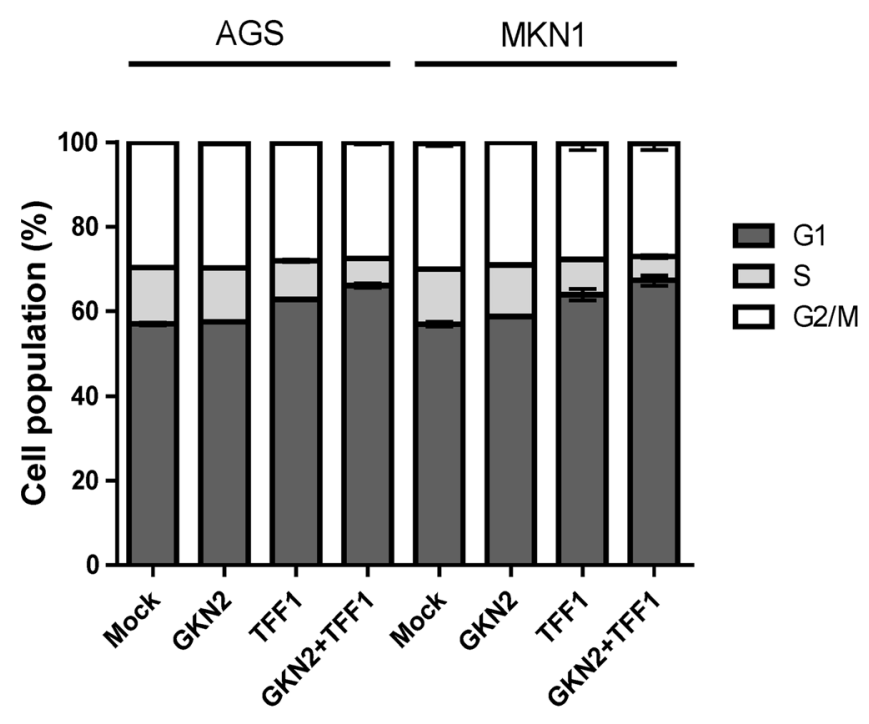

b AGS MKN1

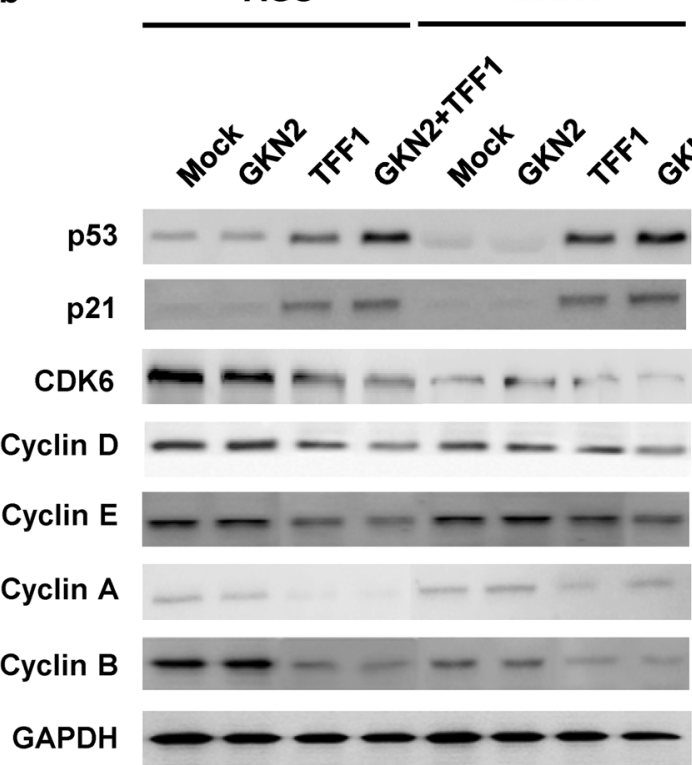

Fig. 2a-b GKN2 and TFF1 synergistically restrict cell cycle progression at the G1/S checkpoint. a In flow cytometric analysis of the cell cycle, TFF1 greatly elevated the percentage of cells at the G1 phase while decreasing the number of cells at the $S$ phase. The interaction of GKN2 and TFF1 induced G1/S cell cycle arrest in both

expression levels of CDK6 and cyclin D, E, A, and B (Fig. 2b). These findings indicate that GKN2 and TFF1 act synergistically to suppress cell cycle progression.

\section{GKN2 and TFF1 synergistically promote apoptosis}

We also examined cell death by staining AGS, MKN1, MKN28, and MKN45 cells with FITC-labeled annexin V and PI. As shown in Fig. 3a and Fig. S2 in the ESM, the percentage of FITC-labeled annexin-V-positive cells was greatly increased after transfection with $T F F 1$ compared to
AGS and MKN1 cells. b GKN2 and TFF1 co-expression significantly increased the expression levels of p53 and p21 but diminished the expression levels of CDK6, cyclins D, E, A, and B compared to GKN2 or TFF1 expression alone

mock-transfected cells $(P<0.05)$. However, ectopic GKN2 expression had no effect on apoptosis. Notably, the formation of the GKN2 and TFF1 complex resulted in significantly more augmentation of the proportion of apoptotic cells compared to the proportions seen with GKN2 or TFF1 alone in AGS, MKN1 MKN28, and MKN45 cells $(P<0.05)$ (Fig. 3a, Supplementary Figure $\mathrm{S} 2$ in the ESM). As expected, ectopic GKN2 expression did not change the level of caspase-3/7 activity (Fig. 3b). However, caspase-3/7 activity was significantly increased in cells transfected with TFFl $(P<0.05)$, and it was even 
more enhanced in cells expressing both GKN2 and TFF1 $(P<0.05)$ (Fig. 3b).

In addition, cleaved forms of caspase- 3 and PARP were present in TFF1-transfected cells. However, there was no change in the expression levels of these proteins in GKN2transfected AGS and MKN1 cells (Fig. 3c). Furthermore, cells transfected with both $G K N 2$ and TFF1 showed significantly elevated expression levels of BAX, cleaved PARP, and cleaved caspase- 3 , whereas the expression levels of anti-apoptotic proteins such as Mcl-1 and Bcl-2 were markedly decreased (Fig. 3c). These findings suggest that GKN2 may acquire pro-apoptotic properties when it is complexed with TFF1, thereby leading to synergistic induction of apoptosis.

\section{Positive correlation between GKN2 and TFF1 in gastric non-tumor and tumor tissues}

We examined the mRNA expression levels of GKN2 and TFF1 in gastric tumor and corresponding non-tumor tissues using real-time RT-PCR. Both GKN2 and TFF1 mRNA expression levels were significantly diminished in tumor tissues compared to those in non-tumor tissues $(P<0.0001)$ (Fig. 4a). Interestingly, the TFF1 mRNA expression level was significantly lower in intestinal-type cancers than in diffuse-type cancers $(P=0.0137)$ (Fig. 4b). However, the GKN2 mRNA expression level was markedly reduced in both histological types of gastric cancer, with no statistically significant difference between the reductions seen in both histological types $(P=0.0970)$ (Fig. 4b). As shown in Fig. 4c, there was a positive correlation between $G K N 2$ and $T F F 1$ mRNA expression in non-tumor samples $(P<0.0001)$. This significant correlation between $G K N 2$ and TFF1 mRNA expression levels was also observed in gastric tumor samples (data not shown).

\section{Expression of TFF1 in gastric cancers}

Immunohistochemical analysis revealed moderate staining for TFF1 mainly in the cytoplasm of gastric mucosal cells (Fig. 5a). A reduction in or loss of TFF1 expression was detected in $10(62.5 \%)$ and $118(77.1 \%)$ of the upper tumors and lower tumors, respectively. According to Lauren's classification [26], TFF1 was expressed in 22 (28.6\%) of 77 intestinal-type gastric cancers and 19 (20.7\%) of 92 diffuse-type gastric cancers (Table 2). However, there was no significant relationship between altered expression of the TFF1 protein and clinicopathologic parameters such as tumor location, tumor size, differentiation, and lymph node metastasis (chi-square test, $P>0.05$, Table 2). When we compared the present data with our previous data [15] on GKN2 expression in these cases, $100(62.5 \%)$ cases were found to show immunonegativity for both GKN2 and TFF1 proteins. There was a borderline-significant correlation between GKN2 and TFF1 expression levels $(P=0.0598)$ (Table 2). Kaplan-Meier analysis revealed that the overall survival rates of gastric cancer patients expressing GKN2 or TFF1 independently did not differ significantly from the overall survival rate of gastric cancer patients co-expressing both GKN2 and TFF1 $(P=0.5204)$ (Fig. 5b).

\section{Discussion}

Three different molecular forms (monomer, dimer, and complex) of TFF1 have been detected in normal human gastric mucosa [27]. Interestingly, the GKN2 protein has been found to be a binding partner for TFF1, and the majority of the TFF1 protein is present as a TFF1:GKN2 heterodimeric complex stabilized by a disulfide bond, suggesting that heterodimer formation may be crucial to TFF1 function [16]. In turn, almost all human GKN2 proteins are present as heterodimers with TFF1. However, GKN2 cannot bind to the other two trefoil proteins TFF2 and TFF3 [28]. Furthermore, it has been confirmed that GKN2 and TFF1 are co-expressed by surface mucus-secreting cells throughout the stomach [17, 28], and that the molecular forms of each of the two proteins are affected by the relative abundance of the other [28]. In the present study, we confirmed the interaction between the GKN2 and TFF1 proteins and the formation of a complex between them in both AGS and MKN1 cells (Fig. 1a).

There is a paucity of data on the ability of GKN2 and TFF1 to regulate cell viability and proliferation in gastric cancers. One study has demonstrated that monomeric TFF1 can reduce cell proliferation but is less potent than its dimeric form [29]. In addition, it has been reported that GKN2 can inhibit the proliferation, migration, and invasion of gastric cancer cells $[19,30]$. Therefore, we took an interest in the effects of the GKN2:TFF1 heterodimer on gastric cancer cell viability and proliferation. Interestingly, GKN2 alone had no significant effect on cell viability and proliferation (Fig. 1b, c, Supplementary Figure S1 in the ESM), whereas TFF1 markedly inhibited both the cell viability and proliferation of AGS, MKN1, MKN28, and MKN45 gastric cancer cells in a time-dependent manner (Fig. 1a, b, Supplementary Figure S1 in the ESM). In particular, the heterodimeric interaction between GKN2 and TFF1 prompted a significantly greater decrease in cell viability and proliferation than GKN2 or TFF1 alone (Fig. 1b, c, Supplementary Figure S1 in the ESM). These results are consistent with those of previous studies reporting that GKN2 alone does not affect cell growth [15] and that $\mathrm{GKN} 2^{-1-}$ mice show no evidence of significant 
a

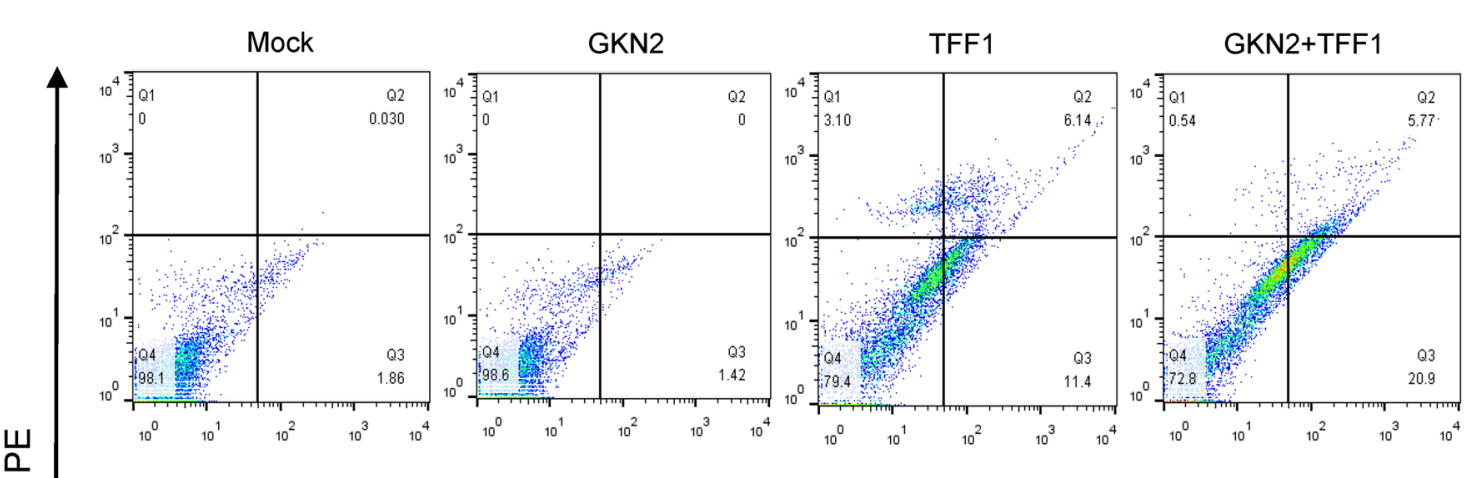

AGS

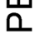

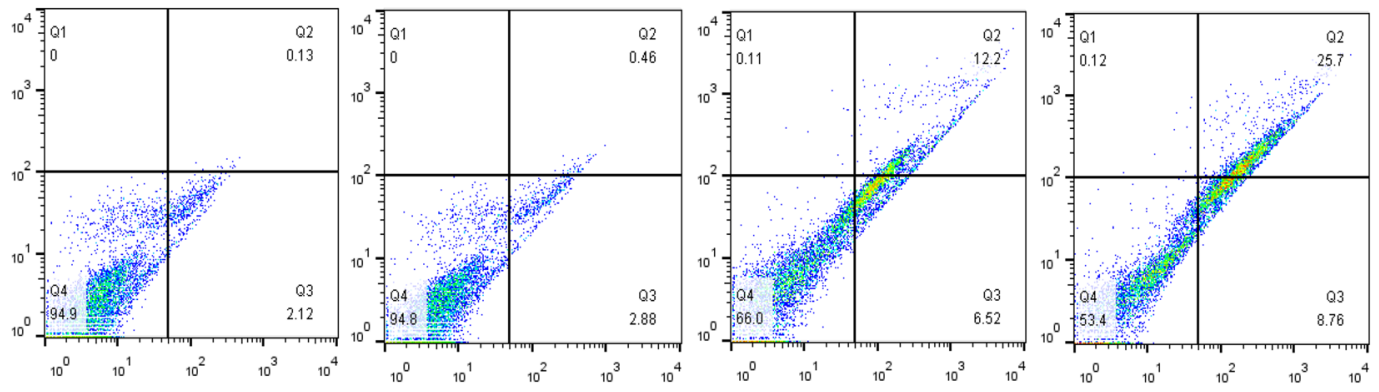

MKN1

FITC

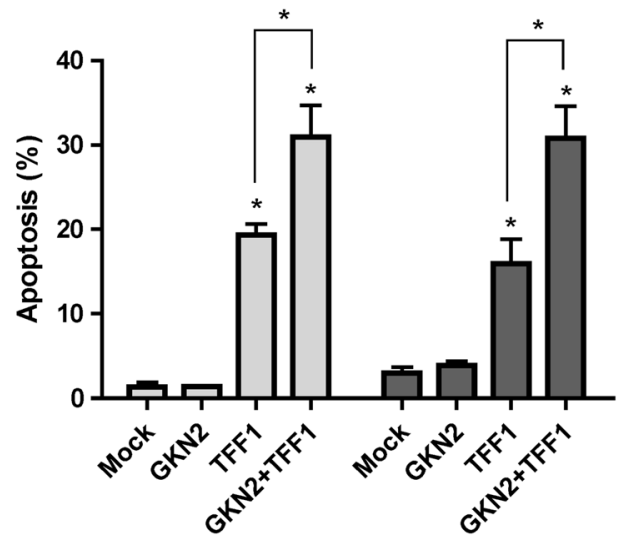

b

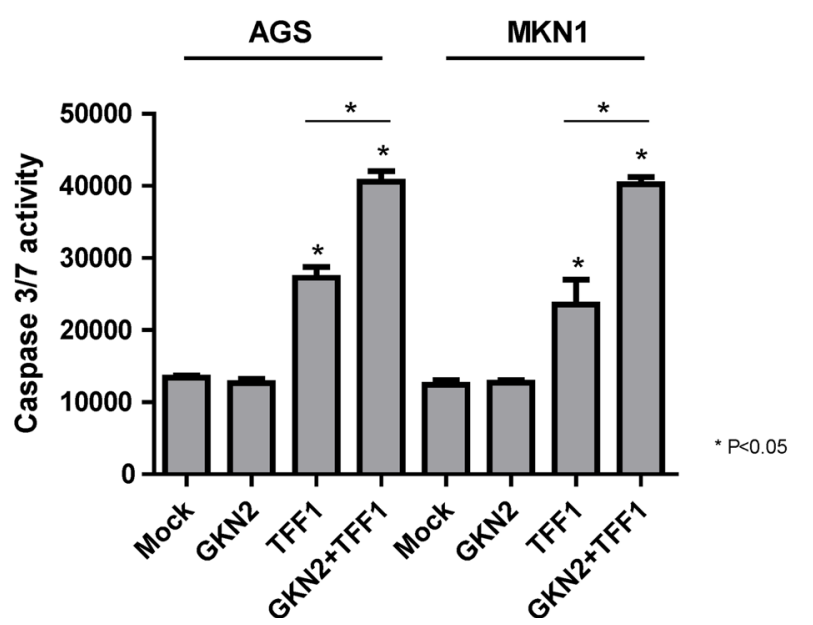

C

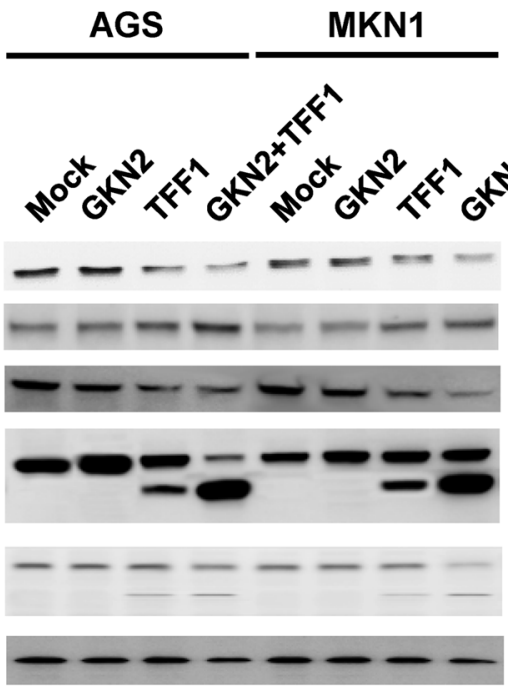


4Fig. 3a-c GKN2 and TFF1 synergistically induce apoptosis. a The annexin-V-binding assay showed a significant increase in the percentage of FITC-labeled annexin-V-positive cells in TFF1-transfected cells. GKN2 and TFF1 co-expression in AGS and MKN1 cells resulted in significantly more augmentation of the proportion of apoptotic cells than GKN2 or TFF1 expression alone. b Caspase-3/7 activity was not changed after transfection with GKN2. However, ectopic TFF1 expression significantly increased caspase-3/7 activity, which was more enhanced in cells expressing both GKN2 and TFF1 than in cells expressing GKN2 or TFF1 alone. c Western blot analysis revealed the presence of cleaved forms of caspase- 3 and PARP in TFF1-transfected cells. Co-expression of GKN2 and TFF1 significantly enhanced the expression levels of BAX, cleaved PARP, and cleaved caspase- 3 proteins while diminishing the expression levels of anti-apoptotic proteins such as Mcl-1 and Bcl-2

epithelial hyperplasia or spontaneous gastric tumors [20]. Thus, GKN2 may be able to acquire growth-inhibiting properties by interacting with TFF1, thereby facilitating the antiproliferative impact of the latter.

Recently, TFF1 has been found to be able to activate p53 by downregulating miR-504 [31]. Another study has reported that TFF1 can inhibit cell proliferation by delaying G1/S cell phase transition while paradoxically increasing the expression of cyclin D1 [32]. To confirm or refute these findings and investigate the possible mechanisms of TFF1- and GKN2-mediated cell growth, the expression of cell cycle regulators was determined and flow cytometric analyses of the cell cycle were performed. The interaction of GKN2 with TFF1 limited cell cycle progression at the G1/S checkpoint (Fig. 2a) and markedly downregulated positive cell cycle regulators while upregulating the expression levels of negative cell cycle regulators (Fig. 2b). These results are consistent with those in previous studies describing the antiproliferative properties of TFF1 [29, 32], suggesting that TFF1 and GKN2 act synergistically to inhibit gastric cancer cell viability and proliferation via G1/S cell cycle arrest and by regulating G0- and G1/S-phase-related proteins.

Interestingly, Bossenmeyer-Pourié et al. have proposed that TFF1 has dual antiproliferative and anti-apoptotic functions: it delays the G1/S cell phase transition and targets the active form of caspase-9 in colon cancer cells [32]. Shi et al. have demonstrated that GKN2 may induce apoptosis by activating Fas, caspase-3, and caspase-8 [33]. In this study, ectopic TFF1 expression was linked to a considerably elevated percentage of apoptotic cells compared to a mock control (Fig. 3a, Supplementary Figure S2 in the ESM). Additionally, TFF1 significantly enhanced caspase-3/7 activity (Fig. 3b) and the expression levels of cleaved PARP, caspase-3, and pro-apoptotic protein Bax, while it suppressed the expression of anti-apoptotic proteins including Mcl-1 and Bcl-2 (Fig. 3c). Most importantly, caspase-3/7 activity (Fig. 3b), the expression of proapoptotic proteins (Fig. 3c), and the proportion of FITC- labeled annexin-V-positive cells (Fig. 3a, SUpplementary Figure S2 in the ESM) were dramatically augmented in AGS, MKN1, MKN28, and MKN45 cells that co-expressed GKN2 and TFF1. These findings suggest that GKN2 and TFF1 can synergistically induce apoptosis in gastric epithelial cells by stimulating caspase-3/7 activity and modulating apoptosis-related proteins.

Notably, Moss et al. revealed that $85 \%$ of diffuse-type and $54 \%$ of intestinal-type gastric cancers show loss of GKN2 expression and that over $70 \%$ of gastric cancers show loss of TFF1 expression [18]. In addition, it has been confirmed that GKN2 is a significant independent predictor of survival in intestinal-type gastric cancers $(P<0.033)$ [18]. In the present study, GKN2 and TFF1 mRNA expression levels were dramatically downregulated or lost in cancer tissues compared to those in corresponding nontumor tissues (Fig. 4a). In addition, the TFF1 mRNA expression level was significantly lower in intestinal-type gastric cancers than in diffuse-type gastric cancers (Fig. 4b). Interestingly, a positive correlation between GKN2 and TFF1 mRNA expression was observed in both adjacent non-tumor tissues and gastric cancer tissues (Fig. 4c). Consistent with this, immunonegativity for both GKN2 and TFF1 proteins was found in 100 cases (62.5\%), and there was a borderline-significant correlation between GKN2 and TFF1 expression (Table 2; Fig. 5a). These results support previous findings that GKN2 and TFF1 are co-expressed in the normal stomach and that such co-expression is frequently disrupted in gastric cancers, suggesting a potential clinical relevance of the GKN2 and TFF1 complex in gastric carcinogenesis. When we examined the overall survival of gastric cancer patients, there was no significant difference between patients who expressed GKN2 or TFF1 alone and those who co-expressed both GKN2 and TFF1 (Fig. 5b). Our findings suggest that attenuated tumor-suppressing activity of TFF1 due to downregulation or loss of GKN2 and TFF1 may play a crucial role early in the development of gastric cancer.

In conclusion, this study presents a novel mechanism of synergistic gastric cancer suppression through the interaction of GKN2 and TFF1. Co-expression of GKN2 and TFF1 massively suppressed the expression of positive cell cycle regulators and induced G1/S arrest, synergistically enhancing the inhibition of cell viability and proliferation. Furthermore, TFF1 increased the expression of negative cell cycle regulators and greatly elevated the proportion of apoptotic cells and caspase-3/7 activity. Finally, we found that the heterodimerization between GKN2 and TFF1 manifested much stronger antiproliferative and pro-apoptotic effects than GKN2 or TFF1 alone, suggesting that GKN2 and TFF1 co-expression may lead to synergistic tumor suppression in gastric cancer. Thus, the combined delivery of functionally synergistic genes such as GKN2 
a

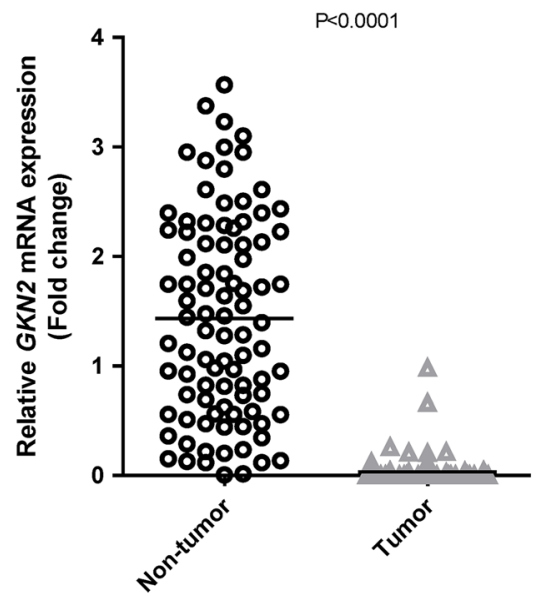

b

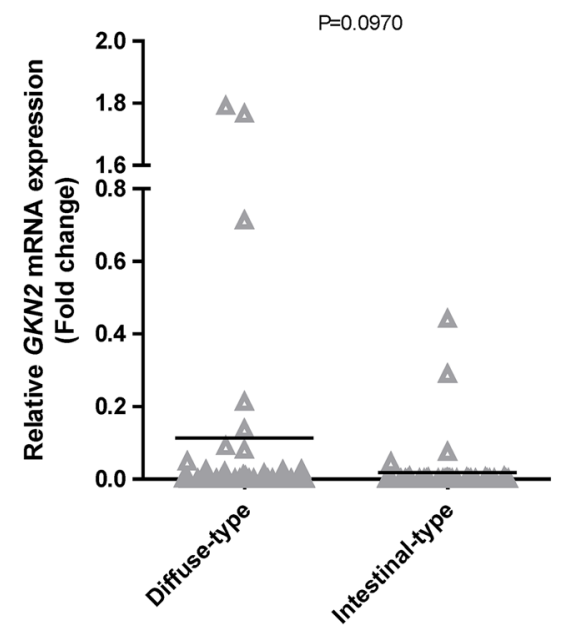

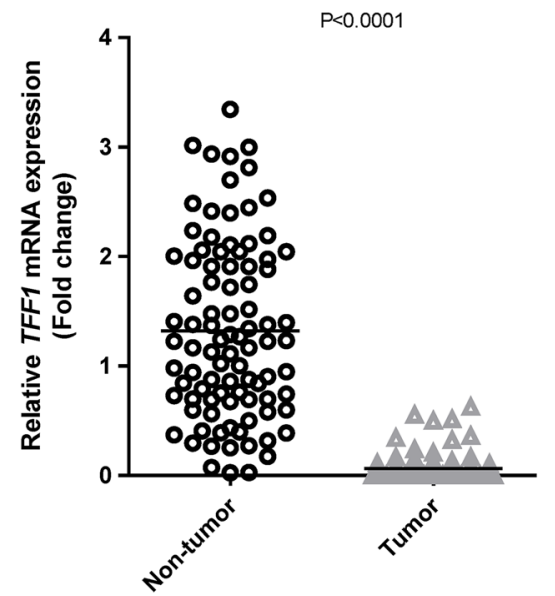

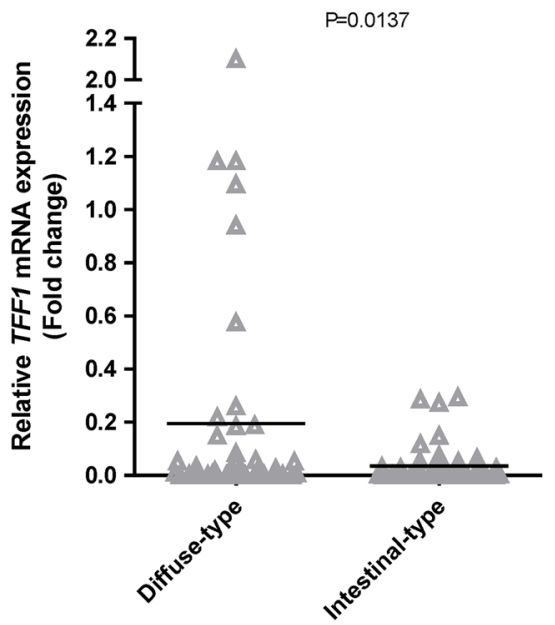

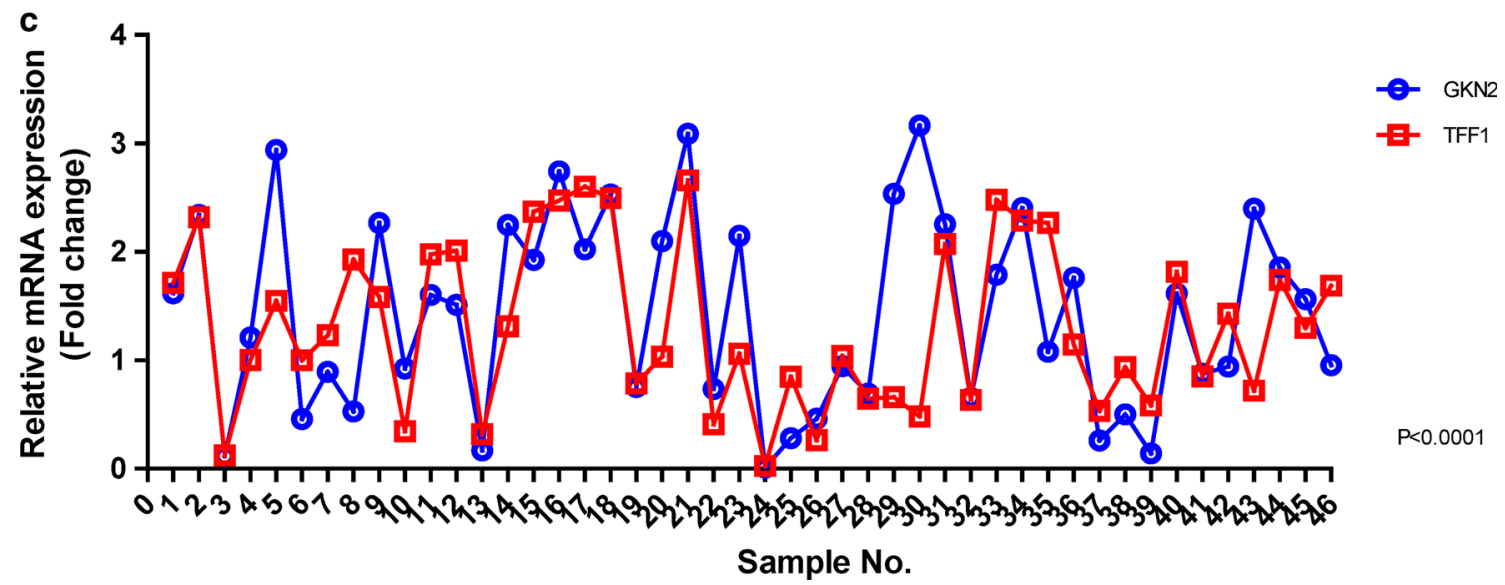

Fig. 4a-c Positive correlation between $G K N 2$ and TFF1 mRNA transcript expression. a In real-time RT-PCR analysis, the mRNA expression levels of $G K N 2$ and $T F F 1$ were greatly decreased in tumor tissues compared to those in corresponding non-tumor samples. b The TFF1 mRNA expression level was significantly lower in intestinaltype gastric cancers. c There was a positive correlation between GKN2 and TFF1 mRNA expression in non-tumor samples. GKN2 and TFF1 mRNA levels were normalized to the mRNA level of the housekeeping gene GAPDH. The standard curve method was used to quantify the relative amounts of gene expression products. This method provides unitless normalized expression values that can be used for direct comparison of the relative amounts of mRNA in different samples. All samples were tested in triplicate. Data are reported as relative quantities according to an internal calibrator using the $2^{-\Delta \Delta \mathrm{CT}}$ method 
Fig. 5a-b TFF1 protein expression in gastric cancers. a Immunostaining showed moderate TFF1 immunopositivity in gastric mucosal epithelial cells (left panel) and gastric cancers (middle panel). Right panel depicts TFF1 immunonegativity found in gastric cancers.

b Kaplan-Meier plots indicating overall survival rates for gastric cancer patients showing an absence of both GKN2 and TFF1 expression, gastric cancer patients showing either GKN2 or TFF1 expression, and gastric cancer patients showing coexpression of GKN2 and TFF1. The $P$ value was determined using the log-rank test a

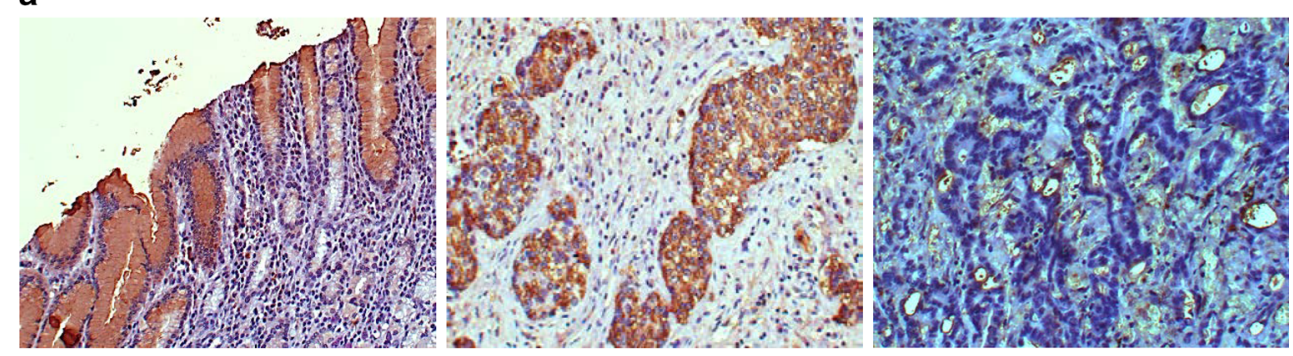

b

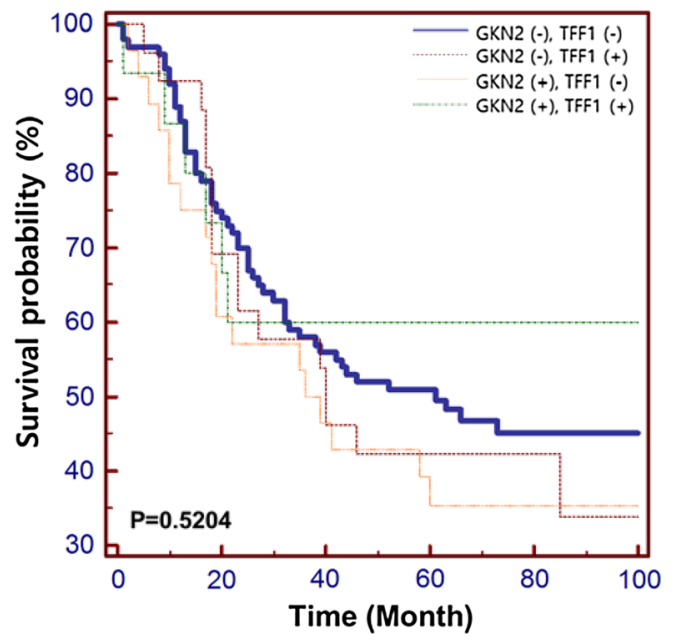

Table 2 Association between TFF1 and GKN2 expression and clinicopathologic parameters in gastric cancers

\begin{tabular}{|c|c|c|c|}
\hline \multirow[t]{2}{*}{ Parameter } & \multicolumn{3}{|c|}{ TFF1 protein expression } \\
\hline & + & - & $P$ value \\
\hline Tumor location & & & 0.1941 \\
\hline Upper & 6 & 10 & \\
\hline Lower & 35 & 118 & \\
\hline Size & & & 0.4888 \\
\hline$<6.5 \mathrm{~cm}$ & 24 & 67 & \\
\hline$>6.5 \mathrm{~cm}$ & 17 & 61 & \\
\hline No. of metastatic L/Ns & & & 0.4709 \\
\hline $\mathrm{N} 1(\geq 1$ and $\leq 2)$ & 5 & 20 & \\
\hline $\mathrm{N} 2(\geq 3$ and $\leq 7)$ & 7 & 31 & \\
\hline N3 $(>7)$ & 29 & 27 & \\
\hline Differentiation $^{a}$ & & & 0.2316 \\
\hline Intestinal & 22 & 55 & \\
\hline Diffuse & 19 & 73 & \\
\hline GKN2 expression & & & 0.0598 \\
\hline+ & 15 & 26 & \\
\hline- & 28 & 100 & \\
\hline
\end{tabular}

$L / N$ lymph node

a Lauren's classification and TFF1 may be a potentially effective therapeutic strategy for gastric cancer.

Acknowledgements This work was supported by the Basic Science Research Program through the National Research Foundation of Korea (NRF) funded by the Ministry of Education, Science and Technology (2015R1A2A2A01002531).

\section{Compliance with ethical standards}

Conflict of interest The authors declare no conflict of interest.

Ethical standards All procedures followed were in accordance with the ethical standards of the responsible committee on human experimentation (institutional and national) and with the Helsinki Declaration of 1964 and later versions.

Informed consent Informed consent was obtained from all patients before they were included in the study.

\section{References}

1. Torre LA, Bray F, Siegel RL, Ferlay J, Lortet-Tieulent J, Jemal A. Global cancer statistics, 2012. CA Cancer J Clin. 2015;65:87-108.

2. Jakowlew SB, Breathnach R, Jeltsch JM, Masiakowski P, Chambon P. Sequence of the pS2 mRNA induced by estrogen in 
the human breast cancer cell line MCF-7. Nucl Acids Res. 1982;10:7895-903.

3. Brown AM, Jeltsch JM, Roberts M, Chambon P. Activation of $\mathrm{pS} 2$ gene transcription is a primary response to estrogen in the human breast cancer cell line MCF-7. Acad Sci USA. 1984;81:6344-8.

4. Rio MC, Bellocq JP, Daniel JY, Tomasetto C, Lathe R, Chenard $\mathrm{MP}$, et al. Breast cancer-associated $\mathrm{pS} 2$ protein: synthesis and secretion by normal stomach mucosa. Science. 1988;241:705-8.

5. Taupin D, Podolsky DK. Trefoil factors: initiators of mucosal healing. Nat Rev Mol Cell Biol. 2003;4(9):721-32.

6. Wong WM, Poulsom R, Wright NA. Trefoil peptides. Gut. 1999;44:890-5.

7. Rio MC, Chenard MP, Wolf C, Marcellin L, Tomasetto C, Lathe R, et al. Induction of $\mathrm{pS} 2$ and hSP genes as markers of mucosal ulceration of the digestive tract. Gastroenterology. 1991;100:375-9.

8. Wright NA, Poulsom R, Stamp G, Van Noorden S, Sarraf C, Elia $\mathrm{G}$, et al. Trefoil peptide gene expression in gastrointestinal epithelial cells in inflammatory bowel disease. Gastroenterology. 1993;104:12-20.

9. Babyatsky MW, deBeaumont M, Thim L, Podolsky DK. Oral trefoil peptides protect against ethanol- and indomethacin-induced gastric injury in rats. Gastroenterology. 1996;110:489-97.

10. Lefebvre O, Chenard MP, Masson R, Linares J, Dierich A, LeMeur M, et al. Gastric mucosa abnormalities and tumorigenesis in mice lacking the pS2 trefoil protein. Science. 1996;274(5285):259-62.

11. Leung WK, Yu J, Chan FK, To KF, Chan MW, Ebert MP, et al. Expression of trefoil peptides (TFF1, TFF2, and TFF3) in gastric carcinomas, intestinal metaplasia, and non-neoplastic gastric tissues. J Pathol. 2002;197:582-8.

12. Park WS, Oh RR, Park JY, Lee JH, Shin MS, Kim HS, et al. Somatic mutations of the trefoil factor family 1 gene in gastric cancer. Gastroenterology. 2000;119(3):691-8.

13. Carvalho R, Kayademir T, Soares P, Canedo P, Sousa S, Oliveira $\mathrm{C}$, et al. Loss of heterozygosity and promoter methylation, but not mutation, may underlie loss of TFF1 in gastric carcinoma. Lab Invest. 2002;82:1319-26.

14. Fujimoto J, Yasui W, Tahara H, Tahara E, Kudo Y, Yokozaki H, et al. DNA hypermethylation at the $\mathrm{pS} 2$ promoter region is associated with early stage of stomach carcinogenesis. Cancer Lett. 2000;149:125-34.

15. Kim O, Yoon JH, Choi WS, Ashktorab H, Smoot DT, Nam SW, et al. GKN2 contributes to the homeostasis of gastric mucosa by inhibiting GKN1 activity. J Cell Physiol. 2014;229(6):762-71.

16. Westley BR, Griffin SM, May FE. Interaction between TFF1, a gastric tumor suppressor trefoil protein, and TFIZ1, a brichos domain-containing protein with homology to SP-C. Biochemistry. 2005;44(22):7967-75.

17. Kouznetsova I, Laubinger W, Kalbacher H, Kalinski T, Meyer F, Roessner A, et al. Biosynthesis of gastrokine-2 in the human gastric mucosa: restricted spatial expression along the antral gland axis and differential interaction with TFF1, TFF2 and mucins. Cell Physiol Biochem. 2007;20(6):899-908.

18. Moss SF, Lee JW, Sabo E, Rubin AK, Rommel J, Westley BR, et al. Decreased expression of gastrokine 1 and the trefoil factor interacting protein TFIZ1/GKN2 in gastric cancer: influence of tumor histology and relationship to prognosis. Clin Cancer Res. 2008;14(13):4161-7.
19. Dai J, Zhang N, Wang J, Chen M, Chen J. Gastrokine-2 is downregulated in gastric cancer and its restoration suppresses gastric tumorigenesis and cancer metastasis. Tumour Biol. 2014;35(5):4199-207.

20. Menheniott TR, O’Connor L, Chionh YT, Däbritz J, Scurr M, Rollo BN, et al. Loss of gastrokine-2 drives premalignant gastric inflammation and tumor progression. $\mathrm{J}$ Clin Invest. 2016;126(4):1383-400.

21. Guang W, Ding H, Czinn SJ, Kim KC, Blanchard TG, Lillehoj EP. Muc1 cell surface mucin attenuates epithelial inflammation in response to a common mucosal pathogen. J Biol Chem. 2010;285:20547-57.

22. Yoon JH, Eun JW, Choi WS, Kim O, Nam SW, Lee JY, et al. NKX6.3 Is a transcription factor for Wnt/ $\beta$-catenin and RhoGTPase signaling-related genes to suppress gastric cancer progression. EBio Med. 2016;9:97-109.

23. Arimura Y, Yano T, Hirano M, Sakamoto Y, Egashira N, Oishi R. Mitochondrial superoxide production contributes to vancomycin-induced renal tubular cell apoptosis. Free Radic Biol Med. 2012;52:1865-73.

24. Walker DG, Link J, Lue LF, Dalsing-Hernandez JE, Boyes BE. Gene expression changes by amyloid b-peptide-stimulated human postmortem brain microglia identify activation of multiple inflammatory processes. J Leukoc Biol. 2006;79:596-610.

25. Kononen J, Bubendorf L, Kallioniemi A, Barlund M, Schraml P, Leighton $\mathrm{S}$, et al. Tissue microarrays for high-throughput molecular profiling of tumor specimens. Nat Med. 1998;4:844-7.

26. Lauren $P$. The two histological main types of gastric carcinoma: diffuse and so-called intestinal-type carcinoma. Acta Pathol Microbiol Scand. 1965;64:31-49.

27. Newton JL, Allen A, Westley BR, May FE. The human trefoil peptide, TFF1, is present in different molecular forms that are intimately associated with mucus in normal stomach. Gut. 2000;46:312-20.

28. May FE, Griffin SM, Westley BR. The trefoil factor interacting protein TFIZ1 binds the trefoil protein TFF1 preferentially in normal gastric mucosal cells but the co-expression of these proteins is deregulated in gastric cancer. Int J Biochem Cell Biol. 2009;41(3):632-40.

29. Calnan DP, Westley BR, May FE, Floyd DN, Marchbank T, Playford RJ. The trefoil peptide TFF1 inhibits the growth of the human gastric adenocarcinoma cell line AGS. J Pathol. 1999; 188(3):312-7.

30. Chu G, Qi S, Yang G, Dou K, Du J, Lu Z. Gastrointestinal tract specific gene GDDR inhibits the progression of gastric cancer in a TFF1 dependent manner. Mol Cell Biochem. 2012;359:369-74.

31. Soutto M, Chen Z, Saleh MA, Katsha A, Zhu S, Zaika A, et al. TFF1 activates p53 through down-regulation of miR-504 in gastric cancer. Oncotarget. 2014;5(14):5663-73.

32. Bossenmeyer-Pourié C, Kannan R, Ribieras S, Wendling C, Stoll I, Thim L, et al. The trefoil factor 1 participates in gastrointestinal cell differentiation by delaying G1-S phase transition and reducing apoptosis. J Cell Biol. 2002;157(5):761-70.

33. Shi LS, Wang H, Wang F, Feng M, Wang M, Guan WX. Effects of gastrokine-2 expression on gastric cancer cell apoptosis by activation of extrinsic apoptotic pathways. Mol Med Rep. 2014;10:2898-904. 